\title{
Sleep in disorders of consciousness: behavioral and polysomnographic recording
}

\author{
Isabella Mertel ${ }^{1,2 \dagger}$, Yuri G. Pavlov ${ }^{1,3^{*}+}$, Christine Barner ${ }^{1}$, Friedemann Müller ${ }^{2}$, Susanne Diekelmann ${ }^{1}$ and
} Boris Kotchoubey ${ }^{1}$

\begin{abstract}
Background: Sleep-wakefulness cycles are an essential diagnostic criterion for disorders of consciousness (DOC), differentiating prolonged DOC from coma. Specific sleep features, like the presence of sleep spindles, are an important marker for the prognosis of recovery from DOC. Based on increasing evidence for a link between sleep and neuronal plasticity, understanding sleep in DOC might facilitate the development of novel methods for rehabilitation. Yet, well-controlled studies of sleep in DOC are lacking. Here, we aimed to quantify, on a reliable evaluation basis, the distribution of behavioral and neurophysiological sleep patterns in DOC over a 24-h period while controlling for environmental factors (by recruiting a group of conscious tetraplegic patients who resided in the same hospital).
\end{abstract}

Methods: We evaluated the distribution of sleep and wakefulness by means of polysomnography (EEG, EOG, EMG) and video recordings in 32 DOC patients (16 unresponsive wakefulness syndrome [UWS], 16 minimally conscious state [MCS]), and 10 clinical control patients with severe tetraplegia. Three independent raters scored the patients' polysomnographic recordings.

Results: All but one patient (UWS) showed behavioral and electrophysiological signs of sleep. Control and MCS patients spent significantly more time in sleep during the night than during daytime, a pattern that was not evident in UWS. DOC patients (particularly UWS) exhibited less REM sleep than control patients. Forty-four percent of UWS patients and 12\% of MCS patients did not have any REM sleep, while all control patients (100\%) showed signs of all sleep stages and sleep spindles. Furthermore, no sleep spindles were found in 62\% of UWS patients and $21 \%$ of MCS patients. In the remaining DOC patients who had spindles, their number and amplitude were significantly lower than in controls.

Conclusions: The distribution of sleep signs in DOC over $24 \mathrm{~h}$ differs significantly from the normal sleepwakefulness pattern. These abnormalities of sleep in DOC are independent of external factors such as severe immobility and hospital environment.

Keywords: Vegetative state, Unresponsive wakefulness, Minimally conscious state, Sleep, Polysomnography, EEG

\footnotetext{
* Correspondence: pavlovug@gmail.com

${ }^{\dagger}$ Isabella Mertel and Yuri G. Pavlov contributed equally to this work.

${ }^{1}$ Institute of Medical Psychology and Behavioral Neurobiology, University of

Tübingen, 72076 Tübingen, Germany

${ }^{3}$ Department of Psychology, Ural Federal University, Ekaterinburg, Russian

Federation 620000

Full list of author information is available at the end of the article
}

(C) The Author(s). 2021, corrected publication March 2021. Open Access This article is licensed under a Creative Commons Attribution 4.0 International License, which permits use, sharing, adaptation, distribution and reproduction in any medium or format, as long as you give appropriate credit to the original author(s) and the source, provide a link to the Creative Commons licence, and indicate if changes were made. The images or other third party material in this article are included in the article's Creative Commons licence, unless indicated otherwise in a credit line to the material. If material is not included in the article's Creative Commons licence and your intended use is not permitted by statutory regulation or exceeds the permitted use, you will need to obtain permission directly from the copyright holder. To view a copy of this licence, visit http://creativecommons.org/licenses/by/4.0/ The Creative Commons Public Domain Dedication waiver (http://creativecommons.org/publicdomain/zero/1.0/) applies to the data made available in this article, unless otherwise stated in a credit line to the data. 


\section{Background}

Acquired brain injury can result in a prolonged state of severe disturbance or even the lack of awareness, referred to as severe disorders of consciousness (DOC). According to the widely accepted definition [1], the presence of the sleep-wakefulness cycle serves as an important symptom distinguishing DOC from acute coma. This alternation of sleep and wakefulness, however, is evaluated only at a behavioral level, that is, as the presence of episodes with open and closed eyes. More recent data indicate that, on the one hand, in some DOC patients, the behavioral sleep-wakefulness cycles do not correspond to any neurophysiological signs of sleep and wakefulness [2]. On the other hand, some acute coma patients can exhibit elements of sleep such as REM sleep, sleep spindles [3], and K-complexes [4].

The two subgroups of DOC are unresponsive wakefulness syndrome (UWS), where patients have reflexive responses but no sign of awareness, and minimally conscious state (MCS), in which patients show unstable conscious behavior but cannot communicate or intentionally use objects. The differential diagnosis between the two is extremely challenging and error-prone [5], which is additionally complicated by strong fluctuations of the arousal level and the associated level of consciousness, particularly in MCS [6], but also in UWS [7], generating variability in the results of repeated behavioral evaluations [8].

Polysomnographic recordings could contribute to the diagnostics of DOC by improving the coordination of task-based diagnostic measurements [9]. Additionally, sleep parameters such as the presence of slow-wave sleep (SWS), rapid eye movement sleep (REM), and sleep spindles may serve as independent markers of the severity of consciousness impairment [10-12]. Some studies even suggest that these sleep parameters may predict the clinical outcome of DOC, i.e., whether or not patients will regain consciousness [13-16].

Although the body of literature about sleep in DOC has substantially increased over the last years, a number of serious issues remain [17]. First, there is no consensus on systematic sleep stage classification in DOC patients $[12,18-21]$. The standard sleep criteria used in healthy individuals $[22,23]$ cannot be applied directly, but have to be adjusted to DOC sleep patterns [20]. To our best knowledge, DOC sleep data are either scored by a single rater, whose blinding is rarely warranted, or a pure automatic analysis is performed. The former substantially decreases the reliability of sleep evaluation, and the latter reduces its validity because the results of the automatic EEG assessment cannot be directly projected onto sleep stages.

Second, many polysomnographic recordings in DOC have been performed during the night $(8-10 \mathrm{~h})$ or an "extended night" interval (16 h) [2, 12, 24]. Thus, sleep of patients who slept during the day might have been incorrectly assessed.

Third, as noted above, it remains unclear whether the "behavioral wakefulness and sleep," used as an important differential diagnostic sign of DOC and measured by the presence of open/closed eyes episodes, really corresponds to "electrophysiological sleep" (derived from polysomnography).

Fourth, none of the studies so far included clinical control groups but only healthy controls. Yet, the living conditions of severely disabled DOC patients radically differ from that of healthy individuals, regarding many parameters that can affect sleep quality, such as the ward room, immobility, the lack of social pressure, and unsolicited disturbance by the hospital personnel.

To solve these issues, in this study, we carried out a reliable expert evaluation of behavioral and electrophysiological sleep over a 24-h period in two groups of DOC patients (UWS and MSC) and a group of tetraplegic control patients.

\section{Methods \\ Patients}

The study was conducted in accordance with the ethical standards of the Declaration of Helsinki and was approved by the Ethics Committee of the Faculty of Medicine, University of Tübingen. The patients and their legal representatives were informed about the study content before study enrollment and gave their written consent. They were informed that participation in the study had no effect on medical treatment and that they can terminate their participation at any time without negative consequences. The study was registered in the German Clinical Study Register (DRKS00009326).

Patients were included according to the following criteria: age between 18 and 69 years (to minimize agedependent effects on sleep [25]), time post ictum from 1 to 24 months, and stable circulation and respiration. Exclusion criteria were as follows: basic EEG activity below $3 \mathrm{~Hz}$; a history of depression, schizophrenia, drug abuse, sleep disorders, or any neurological disorders; and epileptic activity in premorbid EEG recordings. We did not explicitly include extremely severe background EEG patterns (such as isoelectric EEG or burst-suppression pattern) in the list of exclusion criteria because we had not observed such patterns among hospitalized DOC patients in our earlier samples (e.g., [26, 27]). These patterns were not observed in the present sample either. It should be noted that DOC patients in rehabilitation hospitals are not necessarily representative of the entire DOC population. Nevertheless, if patients with these patterns were found in the current study, they would have been excluded. The diagnoses of DOC patients 
were either UWS or MCS. The diagnoses of the clinical control (CC) group patients were Guillain-Barré syndrome or severe high-level spinal cord injury with tetraplegia. Patients were included in the CC group only if their time out of bed did not exceed $4 \mathrm{~h}$ /day.

All patients admitted to the Schön Clinics Bad Aibling (Bavaria, Germany) in the period from February 2016 to February 2018 who fulfilled the above criteria were initially enrolled in the study, resulting in a sample of 19 UWS, 19 MCS, and 12 CC patients. However, two control patients withdrew their consent. Further, six DOC patients were excluded, because their condition worsened $(n=3)$ or improved $(n=3)$ and they did not meet the inclusion criteria anymore. Therefore, data from 16 UWS, $16 \mathrm{MCS}$, and $10 \mathrm{CC}$ patients were recorded and analyzed (see Table 1).

A trained and experienced neurologist repeatedly performed clinical assessment of DOC patients using the Coma Recovery Scale - revised (CRS-r [28]). The last CRS-r evaluation was done on the day before the polysomnographic recording (see Table 1). The UWS and MCS groups did not differ in terms of age (UWS, $46.8 \pm$ 14.6 years; MCS, $48.8 \pm 14.8$ years), gender $(\mathrm{UWS}, \mathrm{m} / \mathrm{f}=$ $7 / 9 ; \mathrm{MCS}, \mathrm{m} / \mathrm{f}=11 / 5)$, time since injury (127 \pm 68.6 days and $114 \pm 46.6$ days, for UWS and MCS, respectively), and the type of injury (traumatic/non-traumatic ratio 6/ 10 and 3/13, for UWS and MCS, respectively). In respect of age and gender, DOC patients also did not significantly differ from the clinical control group patients $(\mathrm{m} /$ $\mathrm{f}=7 / 3$, age $43.7 \pm 18.5$ years).

\section{Recording}

A continuous $24 \mathrm{~h}$ polysomnographic recording included EEG; two channels of electrooculography (EOG), with the electrodes positioned $1 \mathrm{~cm}$ lateral and below and above to the outer canthi of both eyes; three channels of electromyography (EMG), with the electrodes placed on the chin; and video recording. Single cup EEG electrodes were attached at F3, F4, Fz, C3, C4, Cz, P3, P4, Pz, and both mastoids, referenced to $\mathrm{Cz}$ with the ground electrode at Fpz.

The data were recorded using a mobile polysomnography device (SomnoScreenplus, Somnomedics, Germany) with a $256-\mathrm{Hz}$ sampling rate with $0.2-\mathrm{Hz}$ high-pass and $35-\mathrm{Hz}$ low-pass filters.

All recordings were performed in the patients' wards on weekends when no rehabilitative treatments took place. During the recording, all patients (including control patients) had to be moved by nurse personnel to prevent decubitus. After each such intervention, the experimenter checked electrode impedance and renewed electrodes whenever the impedance exceeded $5 \mathrm{k} \Omega$.

The individual medication intake had remained stable during at least 1 week before recording. Recordings started between around 10 am and 12 noon and ended at the same time on the next day.

\section{Data analysis}

The behavioral sleep-wakefulness state of each patient was defined in 30-s intervals based on video recordings. An interval was classified as sleep if the eyes were closed at the onset of the interval and as wakefulness if they were open.

Before the evaluation of electrophysiological sleepwakefulness state, EEG data were re-referenced to averaged mastoids and pre-processed including a notch filter $(50 \mathrm{~Hz})$. The program SchlafAus (developed by Steffen Gais, unpublished) was used for sleep scoring in 30-s intervals. Overall, there are 288030 -s intervals during 24 $\mathrm{h}$, but the number of assessments per recording varied because sometimes more than $24 \mathrm{~h}$ were recorded, and some epochs were lost due to the amplifier's battery replacement (mean number of epochs $=2868, \mathrm{SD}=30.5$, range 2740-2946).

Three independent raters (IM, YGP, CB) scored the data under the supervision of the most experienced fourth person (SD). All raters had previous experience in the classification of sleep stages and were familiar with the essential characteristics of patient groups. Owing to the randomization of patient numbers, all raters were blind to the clinical and demographic patient data.

The first ten recordings were scored by all three raters independently of each other. After having rated two or three patients, they met and discussed their agreements and disagreements. The supervisor defined the main points of discordance to be discussed. At the end of each meeting, scoring criteria, originally based on those of Rechtschaffen and Kales [22], were adapted to maximize a pairwise concordance in each of the three pairs of raters.

After the first ten patients, no further discussion was allowed anymore. The following 32 recordings were randomly distributed among the three pairs of raters. Within each pair, each rater worked independently of the other one. The average percentage agreement (i.e., the number of 30-s epochs on which two raters completely agreed, divided by the total number of epochs evaluated by these two raters and multiplied by 100) for the first ten cases was $83.53 \pm 3.84 \%$, and for subsequent cases, $82.23 \pm 8.06 \%$. In case of conflicting scorings, the raters agreed on the final version where all scoring conflicts were resolved by mutual consensus on an epochby-epoch basis.

The modifications of scoring criteria, as compared with Rechtschaffen and Kales [22], were as follows. Firstly, DOC patients may show local intermittent rhythmic delta activity that might be confused with sleeprelated delta waves. For this reason, nine rather than two 
Table 1 Clinical details of patients

\begin{tabular}{|c|c|c|c|c|c|c|}
\hline ID & Age range (years) & Time since injury (days) & Medication (Sed, Stim, Anticon) & Etiology & EEG grade & CRS-r (A, V, M, OV, C, Ar) \\
\hline UWS1 & $51-55$ & 62 & $0,0,1$ & hypox & 1 & $5(1,0,2,1,0,1)$ \\
\hline UWS2 & $46-50$ & 151 & $1,0,0$ & TBI & 2 & $5(1,0,1,1,0,2)$ \\
\hline UWS3 & $61-65$ & 273 & $0,0,0$ & stroke & 5 & $7(2,1,2,1,0,1)$ \\
\hline UWS4 & $26-30$ & 68 & $0,0,1$ & TBI & 3 & $7(2,1,1,1,0,2)$ \\
\hline UWS5 & $51-55$ & 134 & $1,0,1$ & hypox & 1 & $5(1,0,1,1,0,2)$ \\
\hline UWS6 & $36-40$ & 69 & $0,1,1$ & stroke & 1 & $4(1,0,0,1,0,2)$ \\
\hline UWS7 & $46-50$ & 174 & $2,0,1$ & TBI & 2 & $8(2,1,2,1,0,2)$ \\
\hline UWS8 & $26-30$ & 233 & $1,0,1$ & TBI & 2 & $4(1,0,1,1,0,1)$ \\
\hline UWS9 & $56-60$ & 46 & $0,0,0$ & hypox & 3 & $7(2,1,2,1,0,1)$ \\
\hline UWS10 & $61-65$ & 197 & $1,1,1$ & $\mathrm{SAH}$ & 4 & $6(1,0,2,1,0,2)$ \\
\hline UWS11 & $18-25$ & 134 & $0,0,1$ & $\mathrm{TBI}$ & 3 & $2(0,0,0,1,0,1)$ \\
\hline UWS12 & $41-45$ & 52 & $0,0,2$ & hypox & 3 & $5(1,0,1,2,0,1)$ \\
\hline UWS13 & $56-60$ & 98 & $3,0,1$ & hypox & 1 & $6(2,0,2,1,0,1)$ \\
\hline UWS14 & $56-60$ & 116 & $1,1,0$ & stroke & 3 & $3(0,0,2,1,0,0)$ \\
\hline UWS15 & $18-25$ & 169 & $0,1,0$ & $\mathrm{TBI}$ & 3 & $6(2,0,1,1,0,2)$ \\
\hline UWS16 & $56-60$ & 61 & $0,0,1$ & stroke & 3 & $7(2,0,2,1,0,2)$ \\
\hline MCS1 & $66-70$ & 69 & $1,0,1$ & stroke & 4 & $10(2,2,2,1,1,2)$ \\
\hline MCS2 & $56-60$ & 155 & $0,0,1$ & hypox & 2 & $12(2,1,5,2,1,1)$ \\
\hline MCS3 & $51-55$ & 84 & $1,1,1$ & hypox & 1 & $9(2,0,2,2,1,2)$ \\
\hline MCS4 & $46-50$ & 109 & $0,2,1$ & $\mathrm{TBI}$ & 4 & $10(2,3,2,1,0,2)$ \\
\hline MCS5 & $51-55$ & 60 & $2,0,0$ & $\mathrm{TBI}$ & 5 & $14(3,3,5,1,1,1)$ \\
\hline MCS6 & $51-55$ & 158 & $1,1,1$ & $\mathrm{SAH}$ & 4 & $12(2,3,2,2,1,2)$ \\
\hline MCS7 & $61-65$ & 66 & $0,1,1$ & hypox & 1 & $6(2,2,0,1,0,1)$ \\
\hline MCS8 & $61-65$ & 133 & $0,1,0$ & $\mathrm{SAH}$ & 3 & $9(2,3,2,1,0,1)$ \\
\hline MCS9 & $21-25$ & 150 & $0,1,0$ & hypox & 4 & $9(1,0,5,1,0,2)$ \\
\hline MCS10 & $66-70$ & 140 & $0,1,0$ & TBI & 4 & $16(2,3,5,3,1,2)$ \\
\hline MCS11 & $21-25$ & 234 & $3,0,0$ & enceph & 2 & $8(0,1,4,1,0,2)$ \\
\hline MCS12 & $51-55$ & 92 & $0,0,0$ & $\mathrm{SAH}$ & 5 & $11(2,3,2,2,0,2)$ \\
\hline MCS13 & $18-25$ & 131 & $1,0,0$ & stroke & 3 & $8(1,0,5,1,0,1)$ \\
\hline MCS14 & $41-45$ & 78 & $0,1,1$ & $\mathrm{SAH}$ & 4 & $8(1,2,2,1,0,2)$ \\
\hline MCS15 & $41-45$ & 96 & $1,1,1$ & hypox & 1 & $9(2,2,2,1,0,2)$ \\
\hline MCS16 & $56-60$ & 74 & $1,0,1$ & stroke & 5 & $14(3,1,5,2,1,2)$ \\
\hline $\mathrm{CC} 1$ & $51-55$ & 155 & $1,0,0$ & - & - & - \\
\hline $\mathrm{CC} 2$ & $26-30$ & 1176 & $1,0,2$ & - & - & - \\
\hline $\mathrm{CC} 3$ & $36-40$ & 3876 & $1,0,1$ & - & - & - \\
\hline CC4 & $18-25$ & 379 & $1,0,1$ & - & - & - \\
\hline CC5 & $65-70$ & 33 & $0,0,0$ & - & - & - \\
\hline $\mathrm{CC6}$ & $61-65$ & 267 & $1,0,1$ & - & - & - \\
\hline $\mathrm{CC7}$ & $51-55$ & 131 & $1,0,0$ & - & - & - \\
\hline $\mathrm{CC} 8$ & $66-70$ & 96 & $1,0,1$ & - & - & - \\
\hline CC9 & $26-30$ & 682 & $0,0,0$ & - & - & - \\
\hline $\mathrm{CC} 10$ & $18-25$ & 163 & $1,0,0$ & - & - & - \\
\hline
\end{tabular}

CRS-r total score of Coma Recovery Scale - revised, $A$ auditory, $V$ visual, $M$ motor, OV oromotor/verbal, $C$ communication, $A r$ arousal subscores. Etiology: TBI traumatic brain injury, hypox hypoxic brain injury, enceph encephalitis, $S A H$ subarachnoid hemorrhage. Medication: the number of potentially sleep-affecting medicaments, Sed with muscle relaxation or sedative effects, Stim with stimulating effects, Anticon with anticonvulsive effects (see Additional file 1: Table S1 for a complete list of the medicaments). EEG grade: 1-low amplitude, basic rhythm is difficult to assess; 2-mixture of delta and low theta; 3-stable basic activity in the low-frequency theta domain (4-6 Hz); 4-high-frequency theta mixed with episodes of other rhythms but no desynchronization to visual and verbal stimulation; 5-stable basic activity at 7-8 $\mathrm{Hz}$ OR theta activity responsive to stimulation. Seven of the sixteen MCS patients (patients $4,5,6,8,10,12$ and 16) were MCS+, the other nine were MCS- 
EEG electrodes were used for sleep scoring. This permitted us to detect deviating channels and exclude them from classification.

Secondly, since the EEG amplitude in patients can be influenced by many extracerebral factors, the amplitude criterion for slow waves $(>75 \mu \mathrm{V})$ was not followed. Thirdly, some patients did not show typical markers for sleep stage S2 (sleep spindles, K complexes). In such cases, S2 was assessed on the basis of EEG slowing, the appearance of slow-wave activity, and a further reduction of muscle tone. Slow waves, however, should not cover more than $20 \%$ of the epoch; otherwise, S3 or S4 was scored.

Sleep spindle activity can be visually evaluated, automatically detected, or deducted from the EEG spectrum $[29,30]$. In the literature, there is no agreement on which approach is to be preferred [31-33]. In the present study, we applied both methods: a visual identification of spindles by two independent scorers and, then, an automatic algorithm (with default settings) for sleep spindle quantification [34] to artifact-free stage S2 epochs at $\mathrm{Cz}$ channel. The following statistical analyses involved only patients with at least one clearly present visually detected sleep spindle in the recording. However, Table 2 shows the spindle data (frequency, density, and amplitude) according to the automatic spindle detection in all patients.

Patterns of routine wakefulness EEG were first subdivided into five classes, described in Table 1. Because the corresponding patient subgroups were too small for statistical analyses, classes 1 and 2 were combined into a category of EEG without a clear background rhythmic activity ("bad EEG"), and classes 3, 4, and 5 built together a category of EEG with well-pronounced theta (or low alpha) oscillations ("good EEG"). Similarly, one can see in Table 1 that some etiological groups were small (e.g., only one patient with inflammatory etiology). Therefore, we grouped all etiologies into three categories: traumatic, hypoxic, and others.

Statistical analyses were performed using R v.3.6.0. The 24-h recording was subdivided into day (08.00-20.00) and night (20.00-08.00), according to the time of intensive therapy and lights on versus the time of rest and lights off. A mixed ANOVA included a repeated measures factor DayTime (i.e., day/night) and a between-subject factor Group (UWS, MCS, CC). A similar ANOVA was also performed with the factor Etiology (3 levels: traumatic, hypoxic, others; CC patients excluded). Kruskal-Wallis and Wilcoxon rank sum tests were used for comparing proportions between the groups. When appropriate, we tested the lack of difference between the groups by calculating Bayes factors (BF) using a Bayesian ANOVA or $t$ test. For this analysis, we used the BayesFactor package for $\mathrm{R}$ with default priors.

\section{Results}

Behavioral and electrophysiological sleep

The overall proportion of eyes-closed and eyes-open epochs in the 24-h period was close to 50/50\% (UWS, 49\%; MSC, 51\%; CC, $47 \%$ eyes-closed epochs) and did not differ between all three groups $\left(p=.89, \mathrm{BF}_{01}=200\right)$. Interestingly, the groups differed in the proportion of open and closed eyes epochs during night and daytime (DayTime x Group: $F(2,39)=8.11, p=.001, \eta^{2}=.29$ ). While CC $(t(9)=4.21, p=.0023, d=1.40)$ and MCS $(t(15)=2.41, p=.03, d=0.62)$ patients spent a greater amount of time with closed eyes during the night than daytime, this pattern was not evident in UWS patients $\left(t(15)=0.35, p=.73, d=0.09, \mathrm{BF}_{01}=3.7\right.$; see Fig. 1a).

When electrophysiological sleep data were dichotomized (i.e., 1 -any stage of sleep; 0-wakefulness), the same pattern was observed. CC $(t(9)=6.21, p=.0002$, $d=2.07)$ and MCS $(t(15)=3.04, p=.008, \mathrm{~d}=0.79)$ but not UWS $\left(t(15)=0.76, p=.46, d=0.20, \mathrm{BF}_{01}=3\right)$ patients slept longer during the night than during the day (DayTime $x$ Group: $F(2,39)=8.19, p=.001, \eta^{2}=.30$, see Fig. 1b). Eight of the ten $\mathrm{CC}$ patients took a daytime nap. One UWS patient did not show any signs of electrophysiological sleep despite episodes of closed eyes. All other DOC patients had at least a short interval of sleep during the daytime. One UWS patient slept only during the daytime. Figure 2 shows examples of individual patients' hypnograms.

The sleep of DOC patients appeared to be characterized by frequent changes between sleep and wakefulness. To check this impression, we calculated the number of transitions between sleep and wakefulness (i.e., the intervals that were classified as different from the immediately preceding intervals). The number of transitions did not significantly differ among groups, neither on the basis of behavioral sleep data $(F(2,39)=1.09, p=.35$, $\left.\eta^{2}=.05\right)$ nor when the electrophysiological data were used $\left(F(2,38)=1.46, p=.24, \eta^{2}=.24\right)$. The number of transitions in the electrophysiological sleep data analysis was corrected for the number of sleep epochs because of unequal sleep duration between the patients. One UWS patient who did not sleep at all was excluded from this analysis.

To examine the correspondence between behavioral and electrophysiological data, we calculated the percentage of patients who slept in each of the 2880 epochs (sleep probability). Rank-order correlations between sleep probabilities based on behavioral and electrophysiological data were highly significant in all three groups (rho $=0.87,0.58$, and 0.37 for CC, MCS, and UWS, respectively; all $p$ 's $<0.001$ ). All three correlations were pairwise significantly different from each other, with all $p$ 's $<0.001$. Likewise, the correspondence between behavioral and electrophysiological sleep differed 
Table 2 Sleep characteristics

\begin{tabular}{|c|c|c|c|c|c|c|c|c|}
\hline ID & $\mathrm{S} 1, \mathrm{~min}$ & $\mathrm{~S} 2, \mathrm{~min}$ & SWS, $\min$ & REM, min & $\begin{array}{l}\text { Spindles } \\
\text { present }\end{array}$ & $N$ spindles & $\begin{array}{l}\text { Density, } \\
\text { sp/min }\end{array}$ & $\begin{array}{l}\text { Amplitude, } \\
\mu \mathrm{V}\end{array}$ \\
\hline UWS1 & 88 & 389.5 & 22 & 4.5 & 0 & 19 & 0.06 & 19.57 \\
\hline UWS2 & 33.5 & 434.5 & 31 & 5.5 & 0 & 31 & 0.08 & 14.28 \\
\hline UWS3 & 68.5 & 194 & 86 & 0 & 0 & 0 & 0 & 0 \\
\hline UWS4 & 15.5 & 77.5 & 72.5 & 7.5 & 0 & 0 & 0 & 0 \\
\hline UWS5 & 171 & 189 & 27 & 28.5 & 0 & 10 & 0.06 & 6.86 \\
\hline UWS6 & 0 & 0 & 0 & 0 & 0 & 0 & 0 & 0 \\
\hline UWS7 & 72.5 & 77.5 & 2 & 0 & 0 & 20 & 0.26 & 11.16 \\
\hline UWS8 & 31 & 154 & 242 & 30.5 & 1 & 14 & 0.10 & 9.22 \\
\hline UWS9 & 64 & 258 & 102.5 & 30.5 & 1 & 2 & 0.01 & 7.79 \\
\hline UWS10 & 71 & 3 & 0 & 0 & 0 & 0 & 0 & 0 \\
\hline UWS11 & 2 & 479.5 & 200.5 & 0 & 1 & 89 & 0.21 & 38.29 \\
\hline UWS12 & 46.5 & 190.5 & 52 & 0 & 1 & 125 & 0.68 & 7.13 \\
\hline UWS13 & 49 & 69.5 & 2 & 1.5 & 1 & 7 & 0.15 & 6.99 \\
\hline UWS14 & 81 & 154 & 24.5 & 14 & 0 & 3 & 0.02 & 6.99 \\
\hline UWS15 & 24.5 & 64.5 & 68 & 67.5 & 1 & 65 & 1.13 & 13.62 \\
\hline UWS16 & 13.5 & 78.5 & 204.5 & 0 & 0 & 31 & 0.43 & 15.25 \\
\hline UWS $(M \pm S D)$ & $52 \pm 42.5$ & $175.8 \pm 147.5$ & $71 \pm 78.8$ & $11.9 \pm 18.8$ & & & & \\
\hline MCS1 & 83.5 & 163.5 & 33 & 0 & 0 & 1 & 0.01 & 7.80 \\
\hline MCS2 & 159.5 & 63 & 0 & 86.5 & 0 & 15 & 0.39 & 29.10 \\
\hline MCS3 & 162 & 119.5 & 47 & 7.5 & 0 & 2 & 0.02 & 7.80 \\
\hline MCS4 & 46.5 & 187 & 57 & 19.5 & 0 & 6 & 0.04 & 10.20 \\
\hline MCS5 & 119 & 88.5 & 4 & 24.5 & 1 & 143 & 1.71 & 14.01 \\
\hline MCS6 & 147.5 & 504.5 & 173 & 126 & 1 & 3 & 0.01 & 10.45 \\
\hline MCS7 & 216.5 & 110.5 & 11.5 & 28 & 1 & 8 & 0.08 & 11.71 \\
\hline MCS8 & 67.5 & 373.5 & 63.5 & 10.5 & 1 & 4 & 0.01 & 10.71 \\
\hline MCS9 & 93 & 262 & 75 & 38 & 1 & 120 & 0.48 & 18.64 \\
\hline MCS10 & 75 & 323.5 & 47.5 & 24 & 0 & 5 & 0.03 & 8.32 \\
\hline MCS11 & 4.5 & 295.5 & 253.5 & 90 & 1 & 121 & 0.61 & 8.48 \\
\hline MCS12 & 169 & 104 & 15 & 22 & 1 & 2 & 0.03 & 15.71 \\
\hline MCS13 & 25.5 & 206 & 119.5 & 0 & 1 & 19 & 0.10 & 12.21 \\
\hline MCS14 & 21.5 & 177 & 19.5 & 6 & 1 & 1 & 0.01 & 4.80 \\
\hline MCS15 & 197.5 & 225 & 6.5 & 31.5 & 1 & 323 & 1.48 & 16.48 \\
\hline MCS16 & 34.5 & 194.5 & 0 & 25 & 1 & 11 & 0.06 & 6.60 \\
\hline $\mathrm{MCS}(\mathrm{M} \pm \mathrm{SD})$ & $101.4 \pm 67.2$ & $212.3 \pm 117.3$ & $57.8 \pm 70.3$ & $33.7 \pm 36$ & & & & \\
\hline CC1 & 60 & 231 & 162.5 & 21 & 1 & 209 & 0.99 & 25.37 \\
\hline CC2 & 40 & 191.5 & 144 & 91.5 & 1 & 50 & 0.27 & 25.48 \\
\hline $\mathrm{CC} 3$ & 64.5 & 244.5 & 102 & 83 & 1 & 146 & 0.70 & 20.47 \\
\hline CC4 & 152 & 446.5 & 41 & 49.5 & 1 & 264 & 0.65 & 16.86 \\
\hline CC5 & 111 & 231 & 8.5 & 41 & 1 & 89 & 0.47 & 15.67 \\
\hline CC6 & 56.5 & 209 & 30.5 & 33 & 1 & 20 & 0.11 & 11.42 \\
\hline CC7 & 68.5 & 282.5 & 56 & 17 & 1 & 276 & 1.08 & 15.48 \\
\hline $\mathrm{CC} 8$ & 56 & 286 & 24.5 & 84 & 1 & 130 & 0.52 & 15.77 \\
\hline CC9 & 170.5 & 170.5 & 101 & 88.5 & 1 & 82 & 0.59 & 20.17 \\
\hline
\end{tabular}


Table 2 Sleep characteristics (Continued)

\begin{tabular}{|c|c|c|c|c|c|c|c|c|}
\hline ID & $\mathrm{S} 1, \mathrm{~min}$ & $\mathrm{~S} 2, \mathrm{~min}$ & SWS, min & REM, min & $\begin{array}{l}\text { Spindles } \\
\text { present }\end{array}$ & $N$ spindles & $\begin{array}{l}\text { Density, } \\
\text { sp/min }\end{array}$ & $\begin{array}{l}\text { Amplitude, } \\
\mu \mathrm{V}\end{array}$ \\
\hline $\mathrm{CC} 10$ & 36.5 & 193.5 & 110.5 & 51 & 1 & 281 & 1.53 & 20.50 \\
\hline$C C(M \pm S D)$ & $81.5 \pm 46.8$ & $248.6 \pm 79.1$ & $78 \pm 53.2$ & $56 \pm 28.7$ & & & & \\
\hline
\end{tabular}

UWS unresponsive wakefulness syndrome, MCS minimally conscious state, CC clinical control, S1 sleep stage 1, S2 sleep stage 2, SWS slow-wave sleep, REM rapid eye movement sleep. Spindles present (defined by visual screening): 0 , no; 1 , yes. $N$ spindles, the overall number of spindles; Density, sleep spindle density defined as the overall number of spindles divided by the duration of artifact-free $\mathrm{S} 2$ in minutes; Amplitude, the average amplitude of sleep spindles in $\mu \mathrm{V}$

between the groups when it was analyzed on the basis of single epoch data within each patient $(F(2,38)=4.27$, $\left.p=.021, \eta^{2}=.18\right)$.

\section{Sleep stage distribution}

As shown in Fig. 3, the total amount of sleep as measured with polysomnographic recordings did not significantly differ between the groups $(F(2,39)=2.76, p=.08$, $\eta^{2}=.12$ ), although post hoc tests revealed shorter overall sleep duration in UWS patients compared to the CC group. With regard to single sleep stages, there were significant group differences concerning the time spent in sleep stage $\mathrm{S} 1\left(F(2,39)=3.36, p=.045, \eta^{2}=.15, \mathrm{BF}_{10}=\right.$ $1.8)$ and REM sleep $\left(F(2,39)=7.41, p=.002, \eta^{2}=.28\right.$, $\left.\mathrm{BF}_{10}=19.4\right)$, but not in $\mathrm{S} 2\left(F(2,39)=1.1, p=.34, \eta^{2}=\right.$ $\left..05, \mathrm{BF}_{01}=2.73\right)$ and SWS $\left(F(2,39)=0.28, p=.75, \eta^{2}=\right.$

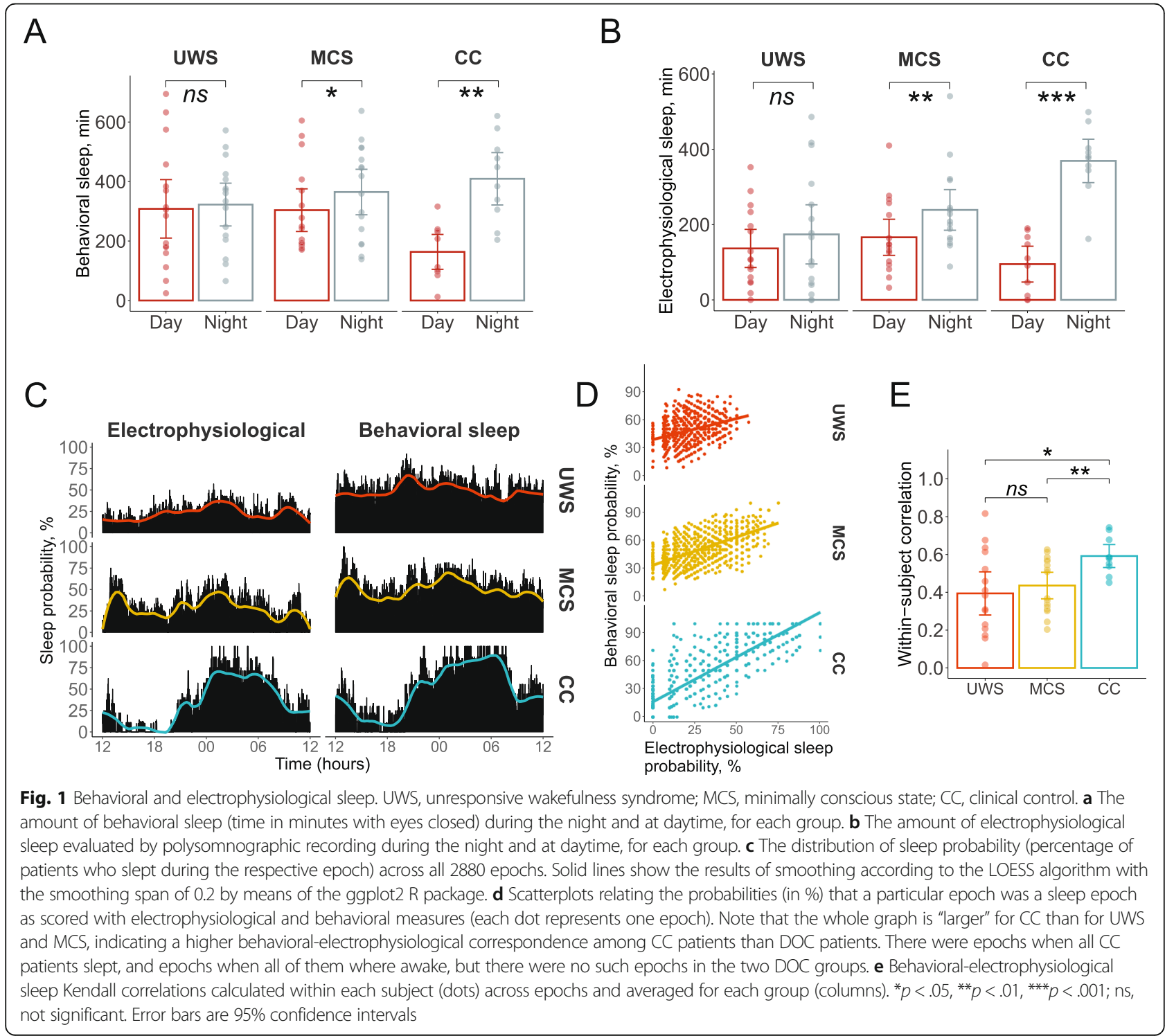




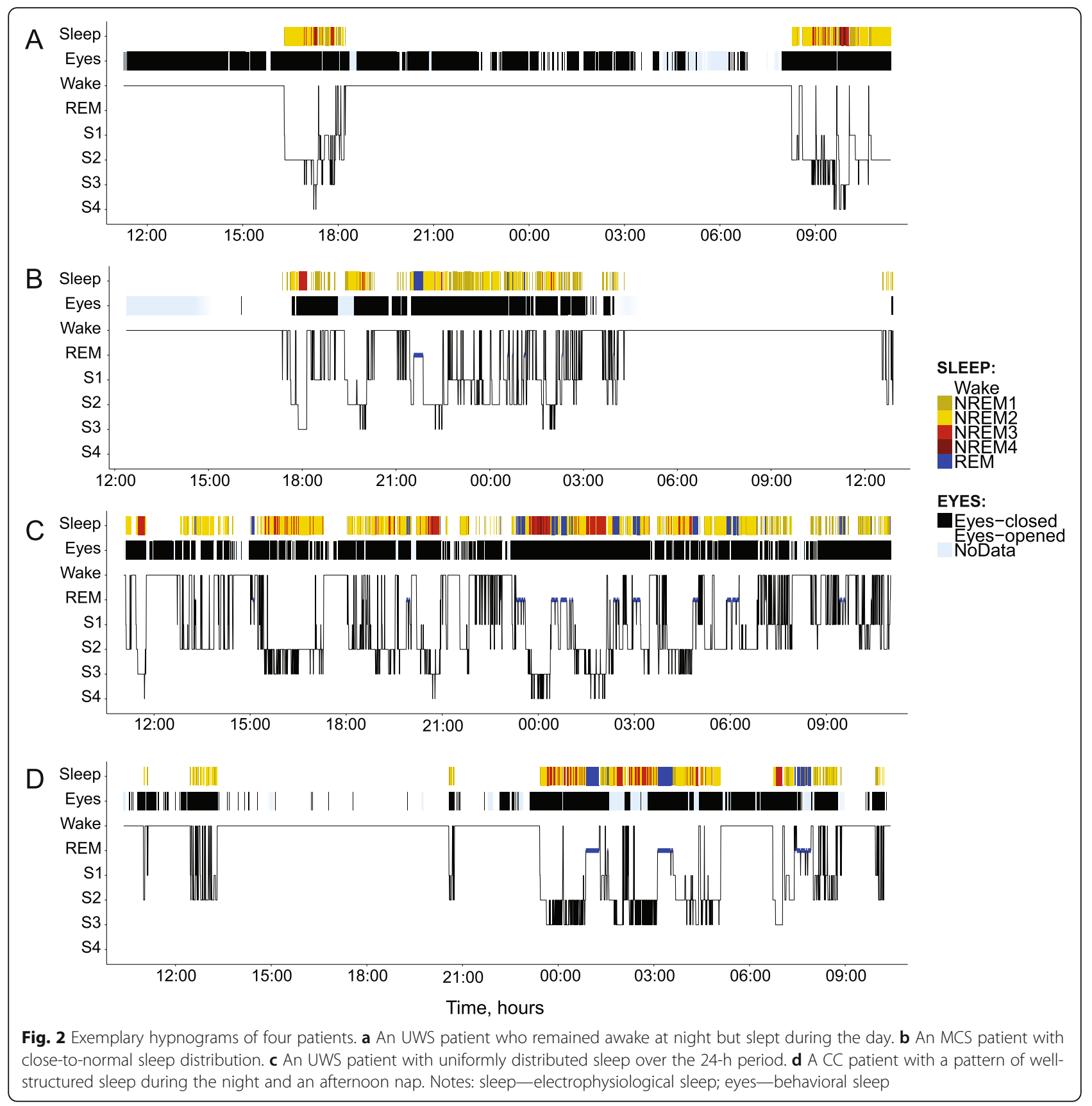

$\left..01, \mathrm{BF}_{01}=4.71\right)$. MCS patients spent more time in S1 than UWS patients $(t(30)=2.49, p=.019, d=0.88)$. Further, UWS patients spent less time in REM sleep than CC patients $(t(24)=4.76, p<.001, d=1.92)$ and MCS $(t(30)=2.15, p=.040, d=0.76)$.

As depicted in Fig. 3d, sleep stages S1 and S2, SWS, and REM sleep were present in all CC patients, but not in all UWS and MCS patients. Statistically, the REM stage was less frequently found in UWS than in MCS $\left(\chi^{2}(1)=3.74, p=.053\right)$ and in CC $\left(\chi^{2}(1)=5.76, p=.016\right)$, but MCS did not differ from CC $\left(\chi^{2}(1)=1.3, p=.25\right)$, resulting in a generally significant Group effect $\left(\chi^{2}(2)=\right.$
8.02, $p=.018$ ). All other sleep stages did not differentiate the groups significantly.

When the diagnostic groups (UWS, MCS) were replaced with etiological groups (traumatic, hypoxic, others; CC patients excluded), hypoxic patients were found to sleep significantly longer in stage S1 (mean = $124.7 \mathrm{~min}$ ) than non-hypoxic patients (means $=46.6 \mathrm{~min}$ and $60.6 \mathrm{~min}$ for TBI and other etiologies, respectively; $\left.F(2,29)=6.28, p=.005, \eta^{2}=.30\right)$. Other sleep stages were not significantly related to etiology $(\mathrm{S} 2: F(2,29)=$ $0.08, p=.92, \eta^{2}<.01$; SWS: $F(2,29)=1.22, p=.31, \eta^{2}=$ .08; REM: $\left.F(2,29)=0.08, p=.92, \eta^{2}<.01\right)$. Likewise, we 


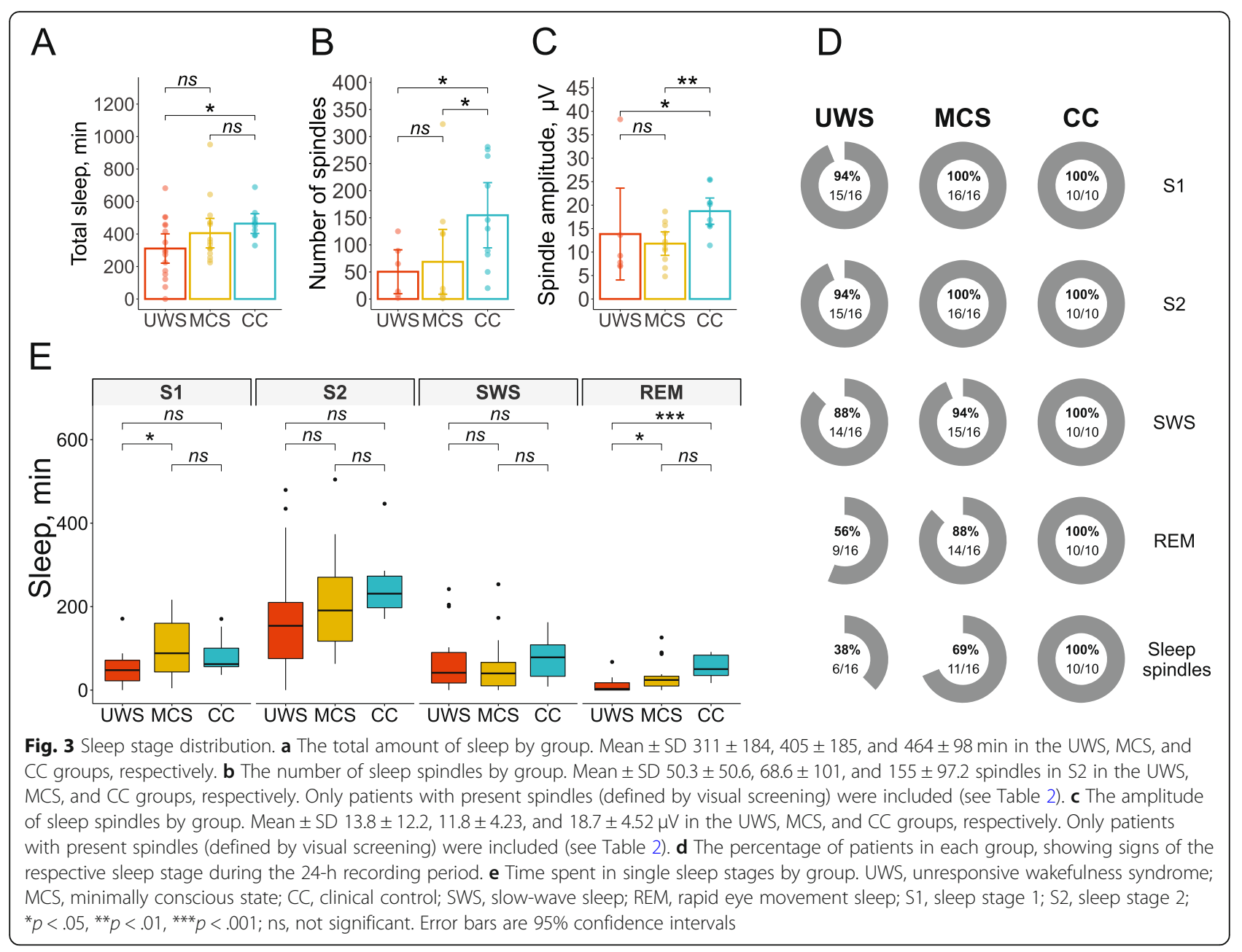

were unable to find any relationship of sleep data with morphological characteristics of brain lesions, possibly because of the difficulty with lesion classification and grouping (see Additional file 1: Table S1).

\section{Effects of age and background EEG}

As it is known that the proportion of SWS and REM sleep decrease with age [25], we tested the possibility that our results were affected by age-related changes in sleep architecture. We confirmed general SWS decrement with age in our sample (Spearman correlation between the amount of SWS and age in years: rho $=-0.44$, $p=.004)$. A correlation of smaller magnitude with age was found for S1 (rho = 0.34, $p=.027$ ) but not for REM sleep $(\mathrm{rho}=-0.18, p=.25)$ or S2 (rho $=-0.1, p=.53$ ). When introducing age as a covariate in our overall sleep stage analysis, the effect of Age was only significant for SWS $\left(F(1,38)=10.75, p=.002, \eta^{2}=.22\right)$. Controlling for age made the effect of Group on SWS even weaker $(F(2$, $\left.38)=0.1, p=.90, \eta^{2}=.005, \mathrm{BF}_{01}=5.29\right)$, while producing negligible effects on REM sleep $(F(2,38)=7.33, p=.002$, $\left.\eta^{2}=.28\right), \mathrm{S} 1 \quad\left(F(2,38)=3.34, p=.05, \eta^{2}=.15\right)$, and S2
$\left(F(2,38)=1.02, p=.37, \eta^{2}=.05\right)$, suggesting that the observed differences between the patient groups were not considerably affected by age.

We further checked whether the background EEG activity, obtained by standard clinical EEG recordings, was related to sleep. None of the sleep stages significantly differed between patients with "good" versus "bad" EEG $\left(\mathrm{S} 1: F(1,30)=2.67, p=.113, \eta^{2}=.08 ; \mathrm{S} 2: F(1,30)=0.30\right.$, $p=.587, \eta^{2}<.01$; SWS: $F(1,30)=0.40, p=.533, \eta^{2}=.01$; REM: $\left.F(1,30)=0.23, p=.632, \eta^{2}<.01\right)$. On the other hand, the background EEG grade significantly depended on etiology ( $p=0.021$, Fisher's exact test). "Bad" EEG characterized seven of the 10 hypoxic patients $(70 \%)$, but only three of the 9 TBI patients (33\%) and two of the 13 patients with vascular and other etiologies (15\%).

\section{Sleep spindles}

Table 2 and Fig. 3d show that the characteristic sleep spindles in S2 were observed in all CC patients but only in a portion of DOC patients, yielding a significant group effect $\left(\chi^{2}(2)=10.44, p=.005\right)$. Specifically, fewer UWS patients (38\%) showed any sleep spindles than CC 
patients $(100 \%)\left(\chi^{2}(1)=9.76, p=.002\right)$; the proportion of MCS patients with identified spindles (69\%) only marginally differed from CC patients $\left(\chi^{2}(1)=3.04, p=.08\right)$ and UWS patients $\left(\chi^{2}(1)=3.72, p=.054\right)$.

To further explore the quality of sleep spindles, only patients with sleep spindles were included, i.e., $n$ $(\mathrm{UWS})=6, n \quad(\mathrm{MCS})=11, n \quad(\mathrm{CC})=10$. Although the average number of spindles significantly differed between the groups $\left(\chi^{2}(2)=6.95, p=.03\right)$, the difference in sleep spindle density did not reach significance $(C C$, $0.689 \pm 0.415$, MCS, $0.415 \pm 0.619$; UWS, $0.382 \pm 0.436$ spindles $\left./ \mathrm{min} ; X^{2}(2)=4.6, p=.1\right)$. CC patients showed a significantly higher number of spindles in sleep stage S2 than UWS patients $(U=87, p=.024)$ and MCS patients $(U=50.5, p=.029)$, whereas the MCS and UWS groups did not differ $(U=31.5, p=.919)$. Similarly, UWS and MCS patients had a lower average spindle amplitude than CC patients $(U=49, p=.042$ and $U=95, p=.004$, respectively), yielding a significant overall group effect $\left(\chi^{2}(2)=8.85, p=.012\right)$.

\section{Discussion}

Previous studies indicated that sleep evaluations are a promising tool in the assessment of DOC patients as the presence of sleep stages seems to be related to the severity of consciousness impairment [10, 11, 13, 35]. Sleep markers can have a higher prognostic value in DOC than many other neurophysiological tests, including event-related brain potentials (ERP) and functional magnetic resonance imaging (fMRI) [14, 36]. Other authors propose a combination of ERP and polysomnography as a predictive tool in DOC [21]. Compared with many other neurophysiological methods, polysomnographic recordings are cost-effective and do not require an immediate response to a stimulus and are, therefore, independent of patient comorbidities (e.g., sensory deficits) and abnormal latency of brain responses.

A number of factors decrease the reliability of previous sleep studies in DOC. Due to numerous abnormalities of EEG and sleep patterns, sleep scoring in DOC is highly challenging. Oscillations in the same frequency range (e.g., $2-3 \mathrm{~Hz}$ ) can indicate both normal SWS and severe brain pathology of DOC patients. Therefore, the mere description of spectral properties and spectrum-based automatic analyzes provide limited insights. The problem is further worsened by the lack of concordant data from independent, blinded raters. In the present study, a group of experienced sleep raters was explicitly trained to adjust Rechtschaffen and Kales' [22] scoring criteria to the particular features of EEG in DOC. Each dataset was independently scored by two raters, who were unaware of the patient's clinical data. The between-rater agreement above $80 \%$ can be regarded as high even for normal sleep [37].
In agreement with previous studies [9, 24, 38, 39], severe abnormalities of circadian rhythms were observed in virtually all DOC patients. Most obviously, the very cyclicity of sleep and wakefulness, i.e., the typical distribution of sleep over the day and night, was severely disturbed. Although there is some evidence that the disturbance may root in a damage or severe dysfunction of the brain stem [40], purely cortical dysfunctions can also yield similar results, particularly when they are broadly spread like the diffuse axonal injury or diffuse gray matter damage after brain hypoxia. Even MCS patients slept less in the night and more during daytime than control patients. Much stronger was this anomaly in UWS patients in which the distribution of sleep and wakefulness did not differ between day and night. Some of the UWS patients did not show night sleep at all but slept only during the day. This observation underlines the necessity of polysomnographic recordings for at least $24 \mathrm{~h}$, because the data of only night sleep can be strongly misleading. Given that a recording day might occasionally be atypical, data collection for a still longer period (48 or $72 \mathrm{~h}$ ) would be useful $[41,42]$, but not at the cost of the decrease of sample size [43].

The presence of numerous sleep episodes during the daytime may account for strong fluctuations observed in the diagnostic behavioral assessment of both MCS [6] and UWS [8]. It is expected that by determining the distribution of periods of decreased arousal level, the risk of diagnostic misjudgment can be reduced.

Although the correlation between behavioral sleep (eyes open-/-closed) and electrophysiological (polysomnographic) signs of sleep was highly significant, it strongly varied among the groups. The correlation was close to 1.0 in control patients, and significantly decreased with the severity of DOC, indicating the possibility of behavioral/electrophysiological sleep dissociation in DOC patients. This finding supports the claim that from the appearance of eyes-open/closed periods in DOC patients, it cannot be directly concluded that the patient is awake or asleep [2].

Whereas all sleep stages were present in all control patients, some sleep stages were entirely lacking in DOC patients. Particularly, significantly fewer UWS patients showed any sign of REM sleep than MCS and control patients. This finding is in line with previous studies, observing greater REM sleep anomalies in UWS than in MCS patients [10, 18, 40, 44]. Other studies reported lower amounts of REM sleep in DOC compared with healthy participants [45]. It has been suggested that disturbances of REM sleep indicate brain stem damage and could thus improve the identification of lesions in neuronal tissues and inform more targeted treatment of individual patients [46]. It could even be speculated that there is a specific relation between REM sleep and 
consciousness: REM sleep deficits might be associated with a lack of dreams, with dreams in turn being peculiar sleep states of consciousness. Furthermore, REM sleep has been proposed to serve the function of preparing the brain for the following state of wakefulness (evidence for this point was reviewed in [47]). It is, however, much too early to speculate about possible causal relationships.

In the present study, DOC patients also showed marked abnormalities with regard to sleep spindles. A total of 15 patients did not show any sleep spindles in S2. Moreover, even the DOC patients who did have identifiable spindles displayed an overall smaller number of spindles and a lower spindle amplitude than control patients. This finding is in line with previous studies that reported sleep spindle deficits in DOC patients $[20,40]$ and greater abnormalities in UWS than in MCS in terms of sleep spindles [44]. Spindle density is generally reduced in severe impairments of cognition and consciousness such as dementia $[48,49]$ and schizophrenia $[50,51]$. But to the best of our knowledge, there are no clinical conditions where sleep spindles are completely absent. This absence may indicate a loss of thalamic circuit integrity [52], which presumably plays a role in the development of DOC [53].

On the background of these multiple abnormalities, there are a number of sleep features for which we did not observe differences between DOC and control patients. The total amount of sleep in control patients was similar to that reported in completely healthy individuals. The amount of sleep in DOC patients was slightly decreased, but this decrease did not reach significance. The average amount of S2 appears to differ between patient groups $(\mathrm{CC}>\mathrm{MCS}>\mathrm{UWS})$, but the intragroup variance in S2 was so large (see Table 2) that the intergroup effect did not become significant. The amount of SWS was virtually identical in all three groups and, moreover, identical to the amount of SWS in normal populations of the corresponding age [25]. In this case, the F-ratio of 0.1 and BF of 5.29 indicate that the lack of significance may be a real null effect and not just a product of insufficient power. If this is true, this finding may indicate that SWS is so important as to maintain its overall stability even under conditions of severe distortions of consciousness and notwithstanding strong fluctuations in the distribution between day and night.

Patients with brain hypoxia were found to stay longer in sleep stage S1 than patients with other etiologies, while S2, SWS, and REM were unrelated to the cause of the DOC. Such a specific effect on S1 was unexpected because scoring of $\mathrm{S} 1$ is particularly difficult even in healthy sleepers. A possible explanation might be, e.g., that a poorly formed, difficult to assess pattern of background wakefulness EEG was observed in 70\% of hypoxic patients but only in $23 \%$ of other patients. Generally, it is easy to confuse S1 with wakefulness state and such mistakes may be expected to be more frequent in patients with worse wakefulness EEG than in patients with well-formed wakefulness EEG. However, we would suggest to wait for a definite interpretation of the result until it has been replicated.

While our findings are largely in line with previous data, there are some differences. In contrast with our results, a large study by Rossi Sebastiano et al. [12] found a significant difference between UWS and MCS in terms of the presence of SWS but not of REM sleep. In a smaller study, Bedini et al. [54] did not observe any differences between UWS and MCS in SWS or in REM sleep. There are at least two important differences between our study and these previous studies. First, it is not quite clear how sleep stages were scored in these previous studies and whether the raters were blind with regard to the patients' diagnosis. Perhaps even more important, Bedini et al. and Rossi Sebastiano et al. performed polysomnographic recordings for less than $24 \mathrm{~h}$. As we have seen that DOC patients, and particularly UWS patients, can show any sleep stage at any time of day/night, it is quite possible that some important sleep epochs were missed during those 5 to $8 \mathrm{~h}$ when the recording was not performed.

A big difficulty in the interpretation of sleep abnormalities in DOC is that the life conditions of such patients differ drastically from those of healthy individuals with respect to many factors, each of which can potentially disturb sleep. DOC patients spend most of their time in bed; during the night, they are regularly disturbed by the light and sounds that cannot be completely avoided in a hospital setting; they are periodically awaked and moved by the personnel to avoid decubitus. Finally, they do not experience the usual social pressure to stay awake during daytime, and the fact that they can sleep almost whenever they want naturally decreases their need for night sleep. These are only a few of numerous external factors of disturbed sleep besides the internal factors related to brain lesions.

Independent studies demonstrated considerable effects of such environmental factors on REM sleep in intensive care unit patients $[55,56]$, but similar studies in DOC patients are entirely lacking. The present study was the first to include a clinical control group that was exposed to the same external environmental factors as the examined DOC patients but did not suffer from conditions of brain damage. Even though this group contained only ten patients, their sleep pattern was radically different from that of DOC patients. Therefore, we conclude that the external factors mentioned above only play a minor, if any, role in the development of massive sleep abnormalities characteristic for DOC. 
The conclusions drawn from the present results are, of course, limited by the moderate size of the sample. Some negative results (e.g., the non-significant findings in the domains of the total amount of sleep and the amount of stage 2) might be different in a double or triple sample. Similarly, our sample size did not permit us to perform subgroup analyses, e.g., a comparison of MCS + versus MCS-. Large multi-center studies would be necessary to investigate such effects, although serious organizational issues remain.

Another limitation, already mentioned above, is that $24 \mathrm{~h}$ is the minimal, but not the optimal recording time. Therefore, an atypical day might eventually have been caught in some patients. Again, however, 48 or $72 \mathrm{~h}$ polysomnographic recordings would present a considerable economical and technical challenge.

We investigated $24 \mathrm{~h}$ distribution of neurophysiological and behavioral correlates of sleep in DOC patients. However, sleep is a complex physiological phenomenon, and a truly comprehensive analysis of patients' circadian rhythms would have to include also peripheral measures such as body temperature, melatonin, and cortisol level.

Like other neurophysiological approaches (e.g., based on EEG, ERP, fMRI, or PET), polysomnography in DOC is still at the stage of investigation and cannot be directly applied as a diagnostic test. The situation is complicated by the lack of an error-free golden standard with which a neurophysiological method can be matched. Large prognostic studies should be conducted to evaluate the usefulness of sleep patterns in the prediction of the clinical outcome, rather than in differentiating the clinical diagnosis. Another potentially important application of sleep studies can be the identification of the individual time window of optimal wakefulness: the optimal time when clinical and electrophysiological assessment should be carried out.

\section{Conclusions}

Our study indicates that the distribution of sleep signs in DOC patients over $24 \mathrm{~h}$ differs significantly from the normal sleep-wakefulness pattern. Specifically, first, DOC patients show severely disturbed circadian rhythms with equally probable sleep occurrence during the day and night in UWS patients; second, eyes-closing behavior is less strongly correlated with neurophysiological sleep patterns; i.e., DOC patients spend longer time with eyes closed while awake than other neurological patients; third, some DOC patients do not have sleep spindles and REM sleep. Notably, these abnormalities of sleep in DOC are due to their brain lesion and cannot be attributed to external factors such as severe immobility and hospital environment.

\section{Supplementary information}

Supplementary information accompanies this paper at https://doi.org/10. 1186/s12916-020-01812-6.

Additional file 1:. Table describing main brain lesions and potentially sleep-affecting medication of the patients.

\section{Acknowledgements}

We thank the project coordinator of the Schoen Clinics for Neurological Rehabilitation Barbara Schäpers, the nurses who collaborated with the scientists, and the patients' families.

\section{Authors' contributions}

BK, IM, YGP, FM, and SD contributed to the conception and design of the study. IM collected the data. YGP, IM, and CB scored the sleep data and supervised by SD. YGP analyzed the data and prepared the figures. IM contributed to the data analysis. IM and YGP drafted the manuscript. BK, FM, $\mathrm{CB}$, and SD brought major revisions in significant proportions of the manuscript. All authors read and approved the final manuscript.

\section{Funding}

The study was supported by the German Research Society (Deutsche Forschungsgemeinschaft, DFG), grant KO-1753/13. Open Access funding enabled and organized by Projekt DEAL.

\section{Availability of data and materials}

The datasets used and/or analyzed during the current study are available from the corresponding author on request.

Ethics approval and consent to participate

The study was conducted in accordance with the ethical standards of the Declaration of Helsinki and was approved by the Ethics Committee of the Faculty of Medicine, University of Tübingen. The patients and their legal representatives were informed about the study content before study enrollment and gave their written consent.

Consent for publication

Not applicable.

\section{Competing interests}

The authors declare that they have no competing interests.

\section{Author details}

${ }^{1}$ Institute of Medical Psychology and Behavioral Neurobiology, University of Tübingen, 72076 Tübingen, Germany. ${ }^{2}$ Schoen Clinics for Neurological Rehabilitation, Bad Aibling, Germany. ${ }^{3}$ Department of Psychology, Ural Federal University, Ekaterinburg, Russian Federation 620000.

Received: 15 June 2020 Accepted: 9 October 2020

Published online: 20 November 2020

References

1. The Multi-Society Task Force on PVS. Medical aspects of the persistent vegetative state. N Engl J Med. 1994;330:1499-508.

2. Landsness E, Bruno M-A, Noirhomme Q, Riedner B, Gosseries O, Schnakers $C$, et al. Electrophysiological correlates of behavioural changes in vigilance in vegetative state and minimally conscious state. Brain. 2011;134:2222-32.

3. Yang X, Song C, Yuan F, Zhao J, Jiang Y, Yang F, et al. Prognostic roles of sleep electroencephalography pattern and circadian rhythm biomarkers in the recovery of consciousness in patients with coma: a prospective cohort study. Sleep Medicine. 2020;69:204-12.

4. Sutter R, Barnes B, Leyva A, Kaplan PW, Geocadin RG. Electroencephalographic sleep elements and outcome in acute encephalopathic patients: a 4-year cohort study. Eur J Neurol. 2014;21:1268-75.

5. Schnakers C, Vanhaudenhuyse A, Giacino J, Ventura M, Boly M, Majerus S, et al. Diagnostic accuracy of the vegetative and minimally conscious state: clinical consensus versus standardized neurobehavioral assessment. BMC Neurol. 2009;9:1

6. Giacino JT, Ashwal S, Childs N, Cranford R, Jennett B, Katz DI, et al. The minimally conscious state definition and diagnostic criteria. Neurology. 2002;58:349-53. 
7. Kotchoubey B, Vogel D, Lang S, Müller F. What kind of consciousness is minimal? Brain Inj. 2014;28:1156-63.

8. Wannez S, Heine L, Thonnard M, Gosseries O, Laureys S. The repetition of behavioral assessments in diagnosis of disorders of consciousness. Ann Neurol. 2017;81:883-9.

9. Bekinschtein TA, Golombek DA, Simonetta SH, Coleman MR, Manes FF. Circadian rhythms in the vegetative state. Brain Inj. 2009;23:915-9.

10. de Biase S, Gigli GL, Lorenzut S, Bianconi C, Sfreddo P, Rossato G, et al. The importance of polysomnography in the evaluation of prolonged disorders of consciousness: sleep recordings more adequately correlate than stimulus-related evoked potentials with patients' clinical status. Sleep Med. 2014;15:393-400.

11. Forgacs PB, Conte MM, Fridman EA, Voss HU, Victor JD, Schiff ND. Preservation of EEG organization in patients with impaired consciousness and imaging-based evidence of command-following. Ann Neurol. 2014;76: 869-79.

12. Rossi Sebastiano D, Visani E, Panzica F, Sattin D, Bersano A, Nigri A, et al. Sleep patterns associated with the severity of impairment in a large cohort of patients with chronic disorders of consciousness. Clin Neurophysiol. 2018; 129:687-93.

13. Kang XG, Li L, Wei $D$, Xu XX, Zhao R, ling YY, et al. Development of a simple score to predict outcome for unresponsive wakefulness syndrome. Crit Care. 2014;18:R37.

14. Kotchoubey B, Pavlov YG. A systematic review and meta-analysis of the relationship between brain data and the outcome in disorders of consciousness. Front Neurol. 2018;9. https://doi.org/10.3389/fneur.2018.00315.

15. Scarpino M, Lolli F, Hakiki B, Atzori T, Lanzo G, Sterpu R, et al. Prognostic value of post-acute EEG in severe disorders of consciousness, using American. Clinical Neurophysiology Society terminology. Neurophysiologie Clinique. 2019:49:317-27.

16. Scarpino M, Lolli F, Hakiki B, Lanzo G, Sterpu R, Atzori T, et al. EEG and coma recovery scale-revised prediction of neurological outcome in disorder of consciousness patients. Acta Neurol Scand. 2020;142:221-8.

17. Kotchoubey B, Pavlov YG. Sleep patterns open the window into disorders of consciousness. Clin Neurophysiol. 2018;129. https://doi.org/10.1016/j.clinph. 2018.01.006.

18. Cologan V, Drouot X, Parapatics S, Delorme A, Gruber G, Moonen G, et al. Sleep in the unresponsive wakefulness syndrome and minimally conscious state. J Neurotrauma. 2013;30:339-46.

19. Wielek T, Lechinger J, Wislowska M, Blume C, Ott P, Wegenkittl S, et al. Sleep in patients with disorders of consciousness characterized by means of machine learning. PLoS One. 2018;13:e0190458.

20. Pavlov YG, Gais S, Müller F, Schönauer M, Schäpers B, Born J, et al. Night sleep in patients with vegetative state. J Sleep Res. 2017;26:629-40.

21. Rossi Sebastiano D, Panzica F, Visani E, Rotondi F, Scaioli V, Leonardi M, et al. Significance of multiple neurophysiological measures in patients with chronic disorders of consciousness. Clin Neurophysiol. 2015;126:558-64.

22. Rechtschaffen A, Kales A. A manual of standardized terminology, techniques and scoring system for sleep stages of human subjects. 1968. http://www. citeulike.org/group/492/article/417041. Accessed 25 Jan 2016.

23. Iber C. The AASM manual for the scoring of sleep and associated events: rules, terminology and technical specifications. American Academy of Sleep Medicine; 2007

24. Wislowska M, del Giudice R, Lechinger J, Wielek T, Heib DPJ, Pitiot A, et al. Night and day variations of sleep in patients with disorders of consciousness. Sci Rep. 2017;7. https://doi.org/10.1038/s41598-017-00323-4.

25. Ohayon MM, Carskadon MA, Guilleminault C, Vitiello MV. Meta-analysis of quantitative sleep parameters from childhood to old age in healthy individuals: developing normative sleep values across the human lifespan. Sleep. 2004;27:1255-73.

26. Kotchoubey B, Lang S, Mezger G, Schmalohr D, Schneck M, Semmler A, et al. Information processing in severe disorders of consciousness: vegetative state and minimally conscious state. Clin Neurophysiol. 2005;1 16:2441-53.

27. Yu T, Lang S, Vogel D, Markl A, Muller F, Kotchoubey B. Patients with unresponsive wakefulness syndrome respond to the pain cries of other people. Neurology. 2013;80:345-52.

28. Giacino JT, Kalmar K, Whyte J. The JFK Coma Recovery Scale-Revised: measurement characteristics and diagnostic utility. Arch Phys Med Rehabil. 2004;85:2020-9.

29. De Gennaro L, Ferrara M. Sleep spindles: an overview. Sleep Med Rev. 2003: 7:423-40.
30. O'Reilly C, Nielsen T. Automatic sleep spindle detection: benchmarking with fine temporal resolution using open science tools. Front Hum Neurosci. 2015;9. doi:10/ggrbkh.

31. Huupponen E, Gómez-Herrero G, Saastamoinen A, Värri A, Hasan J, Himanen S-L. Development and comparison of four sleep spindle detection methods. Artif Intell Med. 2007:40:157-70.

32. Lacourse K, Delfrate J, Beaudry J, Peppard P, Warby SC. A sleep spindle detection algorithm that emulates human expert spindle scoring. J Neurosci Methods. 2018. https://doi.org/10.1016/j.jneumeth.2018.08.014.

33. Warby SC, Wendt SL, Welinder P, Munk EGS, Carrillo O, Sorensen HBD, et al. Sleep-spindle detection: crowdsourcing and evaluating performance of experts, non-experts and automated methods. Nat Methods. 2014:11:385-92.

34. Muehlroth BE, Werkle-Bergner M. Understanding the interplay of sleep and aging: methodological challenges. Psychophysiology. 2020;57. https://doi. org/10.1111/psyp.13523.

35. Avantaggiato P, Molteni E, Formica F, Gigli GL, Valente M, Lorenzut S, et al. Polysomnographic sleep patterns in children and adolescents in unresponsive wakefulness syndrome. J Head Trauma Rehabil. 2015;30: $334-46$.

36. Arnaldi D, Terzaghi M, Cremascoli R, De Carli F, Maggioni G, Pistarini C, et al. The prognostic value of sleep patterns in disorders of consciousness in the sub-acute phase. Clin Neurophysiol. 2015. https:// doi.org/10.1016/j.clinph.2015.10.042.

37. Danker-Hopfe H, Kunz D, Gruber G, Klösch G, Lorenzo JL, Himanen SL, et al. Interrater reliability between scorers from eight European sleep laboratories in subjects with different sleep disorders. J Sleep Res. 2004;13:63-9.

38. Blume C, Lechinger J, Santhi N, del Giudice R, Gnjezda M-T, Pichler G, et al. Significance of circadian rhythms in severely brain-injured patients: a clue to consciousness? Neurology. 2017;88:1933-41.

39. Cruse D, Thibaut A, Demertzi A, Nantes JC, Bruno M-A, Gosseries O, et al. Actigraphy assessments of circadian sleep-wake cycles in the vegetative and minimally conscious states. BMC Med. 2013;11:18.

40. Isono M, Wakabayashi Y, Kamida T, Kobayashi H. Sleep cycle in patients in a state of permanent unconsciousness. Brain Inj. 2002;16:705-12.

41. Schabus M, Wislowska M, Angerer M, Blume C. Sleep and circadian rhythms in severely brain-injured patients - A comment. Clinical Neurophysiology. 2018;129:1780-4.

42. Blume C, Angerer M, Raml M, del Giudice R, Santhi N, Pichler G, et al. Healthier rhythm, healthier brain? Integrity of circadian melatonin and temperature rhythms relates to the clinical state of brain-injured patients. Eur J Neurol. 2019. https://doi.org/10.1111/ene.13935.

43. Schabus M, Wislowska M, Angerer M, Blume C. Sleep and circadian rhythms in severely brain-injured patients - A comment. Clin Neurophysiol. 2018;129: 1780-4.

44. Aricò I, Naro A, Pisani LR, Leo A, Muscarà N, Salvo SD, et al. Could combined sleep and pain evaluation be useful in the diagnosis of disorders of consciousness (DOC)? Preliminary findings. Brain Injury. 2016;30:159-63.

45. Oksenberg A, Gordon C, Arons E, Sazbon L. Phasic activities of rapid eye movement sleep in vegetative state patients. Sleep. 2001;24:703.

46. Cologan V, Schabus M, Ledoux D, Moonen G, Maquet P, Laureys S. Sleep in disorders of consciousness. Sleep Med Rev. 2010;14:97-105.

47. Peever J, Fuller PM. The biology of REM sleep. Curr Biol. 2017;27:R1237-48.

48. Ktonas PY, Golemati S, Xanthopoulos P, Sakkalis V, Ortigueira MD, Tsekou H, et al. Time-frequency analysis methods to quantify the time-varying microstructure of sleep EEG spindles: possibility for dementia biomarkers? J Neurosci Methods. 2009;185:133-42.

49. Scullin MK, Bliwise DL. Sleep, cognition, and normal aging: integrating a half century of multidisciplinary research. Perspect Psychol Sci. 2015;10:97-137.

50. Manoach DS, Pan JQ, Purcell SM, Stickgold R. Reduced sleep spindles in schizophrenia: a treatable endophenotype that links risk genes to impaired cognition? Biol Psychiatry. 2016;80:599-608.

51. Wamsley EJ, Tucker MA, Shinn AK, Ono KE, McKinley SK, Ely AV, et al. Reduced sleep spindles and spindle coherence in schizophrenia: mechanisms of impaired memory consolidation? Biol Psychiatry. 2012; 71:154-61.

52. Fernandez LMJ, Lüthi A. Sleep spindles: mechanisms and functions. Physiol Rev. 2020;100:805-68.

53. Giacino JT, Fins JJ, Laureys S, Schiff ND. Disorders of consciousness after acquired brain injury: the state of the science. Nat Rev Neurol. 2014;10:99-114 
54. Bedini G, Bersano A, Sebastiano DR, Sattin D, Ciaraffa F, Tosetti V, et al. Is Period3 Genotype Associated With Sleep and Recovery in Patients With Disorders of Consciousness? Neurorehabil Neural Repair. 2016;30:461-9.

55. Huang H-W, Zheng B-L, Jiang L, Lin Z-T, Zhang G-B, Shen L, et al. Effect of oral melatonin and wearing earplugs and eye masks on nocturnal sleep in healthy subjects in a simulated intensive care unit environment: which might be a more promising strategy for ICU sleep deprivation? Crit Care. 2015:19:124.

56. Lewis SR, Schofield-Robinson OJ, Alderson P, Smith AF. Propofol for the promotion of sleep in adults in the intensive care unit. Cochrane Database Syst Rev. 2018. https://doi.org/10.1002/14651858.cd012454.pub2.

\section{Publisher's Note}

Springer Nature remains neutral with regard to jurisdictional claims in published maps and institutional affiliations.

Ready to submit your research? Choose BMC and benefit from:

- fast, convenient online submission

- thorough peer review by experienced researchers in your field

- rapid publication on acceptance

- support for research data, including large and complex data types

- gold Open Access which fosters wider collaboration and increased citations

- maximum visibility for your research: over $100 \mathrm{M}$ website views per year

At BMC, research is always in progress.

Learn more biomedcentral.com/submissions 